Research Article

\title{
Preconception Care Uptake and Immediate Outcomes among Discordant Couples Accessing Routine HIV Care in Kenya
}

\author{
Nyawira Gitahi $\mathbb{D}^{1},{ }^{1}$ Sheila Juliet Eshiwani $\mathbb{D}^{3},{ }^{3}$ Kenneth Mutai, ${ }^{3}$ Jared Ongechi Mecha $\mathbb{D}^{\circ}{ }^{2}$ \\ and James Njogu Kiarie ${ }^{4}$ \\ ${ }^{1}$ Institute of Tropical Medicine and Infectious Disease (ITROMID), Kenya Medical Research Institute, Nairobi, Kenya \\ ${ }^{2}$ Department of Clinical Medicine and Therapeutics, University of Nairobi, Nairobi, Kenya \\ ${ }^{3}$ Kenyatta National Hospital, Nairobi, Kenya \\ ${ }^{4}$ Department of Obstetrics and Gynaecology, University of Nairobi, Nairobi, Kenya
}

Correspondence should be addressed to Nyawira Gitahi; gnyawira@gmail.com

Received 13 March 2019; Revised 16 January 2020; Accepted 23 March 2020; Published 9 June 2020

Academic Editor: Peter E. Schwartz

Copyright (c) 2020 Nyawira Gitahi et al. This is an open access article distributed under the Creative Commons Attribution License, which permits unrestricted use, distribution, and reproduction in any medium, provided the original work is properly cited.

Introduction. A large proportion of new HIV infections occur within discordant partnerships making discordance a significant contributor to new HIV infections in Africa. Despite the role of preconception care for HIV discordant couples, there is little data on fertility desire and preconception care uptake. This study aimed at documenting fertility desire (desire to conceive), determining the uptake of preconception care, identifying HIV prevention strategies used during preconception care, and determining immediate conception outcomes among HIV discordant couples in Kenya. Methods. We retrospectively extracted electronic medical record data on discordant couples at an HIV care discordant couples' clinic. We included data on couples who expressed a desire to conceive and were offered preconception care and followed up for 29 months. We collected data on sociodemographic characteristics, preconception prevention methods, and associated outcomes. Results. Among couples, with male HIV-positive partners, there was a twofold likelihood of accepting preconception services $(\mathrm{OR}=2.3$, CI 95\% $(1,1,5.0))$. A shorter discordant union was independently associated with the uptake of preconception services $(\mathrm{OR}=0.92$, CI $95 \%(0.86,0.98))$. The most used prevention intervention (38.5\%) among discordant couples was a combination of pre-exposure prophylaxis (PrEP) by the uninfected partner, alongside HAART by the partner living with HIV. Pregnancy rates did not significantly $(p=0.06)$ differ among those who took up preconception care versus those who did not. HIV-negative partners of couples who declined preconception care had a significantly $(p=0.04)$ higher attrition from clinic follow-up. One confirmed seroconversion occurred; an HIV incidence rate of 0.19 per 100 person-years.Conclusion. The study demonstrates the feasibility of implementing safe and effective preconception servicesas part of routine HIV care for discordant couples living in low resource settings. The provision and the utilisation of safer conception services may be hindered by the poor retention to follow-up and care of HIV-negative partners. This challenge may impede the expected benefits of preconception care as an HIV prevention intervention.

\section{Introduction}

In high HIV prevalence settings, up to $50 \%$ of the new infections occur within HIV discordant partnerships [1-3]. HIV serodiscordant couples desiring children can knowingly risk HIV transmission with unprotected intercourse $[1,2,4,5]$. In this regard, preconception care among discordant couples is urgent and critical both in the prevention of HIV transmission to the uninfected partner and also in the prevention of vertical transmission from mother to baby [6].

Among persons living with HIV (PLWHIV), the goals of preconception care include facilitating reproductive decision-making and minimising risks of horizontal and vertical HIV transmission [6, 7]. There are existing recommended prevention interventions such as highly active antiretroviral 
therapy (HAART) for all positive partners, preconception counselling, condom provision, and emphasis on planned pregnancies towards safer conception among discordant couples [6]. Additionally, the use of pre-exposure prophylaxis, artificial vaginal insemination, timed unprotected intercourse, and sperm wash have been described in the literature [8-12]. The recommended World Health Organization (WHO) preconception care package for all couples [6] includes among others, vaccination against preventable diseases, assessment for sexually transmitted infections (STIs), and nutritional and genetic assessment. There is existing data that PLWHIV who are adherent to highly active antiretroviral therapy (HAART) and maintaining an undetectable viral load have effectively no risk of sexually transmitting HIV [7, 13-15]. A 96\% reduction in HIV transmission among discordant couples [13] even in the presence of inconsistent condom use [16] and over long periods (up to 10 years) has been reported. Moreover, maternal viral suppression to undetectable levels before conception, during pregnancy, and at the time of delivery makes perinatal HIV transmission almost entirely preventable $[17,18]$. Given these apparent benefits, the WHO now recommends preconception care as a standard component of primary care for HIV discordant couples [6].

The uptake of safer conception service in high-burden, resource-limited settings remains mostly unknown [10]. In Kenya, $50 \%$ of persons with known HIV status are in discordant partnerships [19]. Globally and in sub-Saharan Africa, most of the data available on HIV prevention and conception are primarily from research settings $[2,3,15,17,20]$. Specifically, data gaps exist on the feasibility of the implementation of safer conception models in lowand middle-income settings [15, 21, 22].

We report on fertility desire (desire to conceive) among HIV discordant couples, uptake of preconception care, HIV prevention preferred methods, and immediate conception outcomes and couple retention outcomes among HIV discordant couples in sub-Saharan Africa.

\section{Methods}

2.1. Study Design. This study utilises data from a cohort of HIV discordant couples enrolled in care from April 2013 to September 2015.

2.2. Study Setting. We conducted this study in a public referral and teaching hospital that offers free HIV care within a clinic that caters exclusively to discordant couples. Enrolment criteria to the discordant clinic were HIV serodiscordant, age above 18 years, willingness to receive care as a couple, discordant relationship existent for at least three months, and residing within proximity to the clinic or able to attend three monthly visits.

Within the discordant couples' clinic, interventions directly targeting discordant couples were provided as per the $2015 \mathrm{WHO}$ guidelines; HAART to all HIV positive partners and routine viral load monitoring [14]. Services offered to the HIV-negative partner include quarterly HIV testing, STI screening and treatment, condom and lubricant provision, and risk reduction counselling [4]. All couples who reported that they did not desire to conceive in the next six months were provided with contraception counselling and offered or referred for the contraception option of choice. We explored fertility intention as part of standard care of treatment within the clinic, usually at every visit through the question "Do you plan to conceive within the next three months?" We used routinely collected data within the electronic medical patient encounter forms.

2.3. Preconception-Specific Services. Couples who expressed a desire to conceive and where the HIV-positive person had achieved viral suppression were enrolled to preconception care sessions. These scheduled monthly sessions, included discussions on safer conception practices and the importance of optimum viral suppression $(<1000$ copies $/ \mathrm{ml})$. Preconception interventions discussed were PrEP and timed unprotected intercourse (TUI) during ovulation. Clinic staff also demonstrated self-artificial vaginal insemination (AVI). The preconception care clinic team consisted of a general practitioner medical doctor, a nurse trained in reproductive health care, and a gynaecologist. Preconception care required the couple to attend the clinic every month until conception occurred for at least one year. Failure to conceive after one year of follow-up resulted in a referral for gynaecological management and further clinical evaluation.

We extracted data for this study from routinely collected electronic medical records during the provision of care discordant couples' clinic between April 2013 and September 2015. In this study, we only analysed data from couples enrolled for HIV care at the discordant couples' clinic who expressed a desire to conceive during routine visits included analysis.

We obtained ethical approval from the Kenyatta National Hospital/University of Nairobi joint Ethical and Scientific Committee for the use of routine programmatic data. The outcome measures were uptake of preconception care services, uptake of HIV prevention methods, conception outcomes, and discordant couple retention to care. We also examined sociodemographic characteristics and seroconversion events.

Data were analysed using SPPS version 23, IBM Corporation, Armonk, New York. Means and standard deviations (SD), medians and interquartile ranges (IQR), and proportions summarised the continuous and categorical variables, respectively. Chi-square and Mann-Whitney $U$ tests were used for comparisons between those who took up preconception care versus those who did not and to compare those who conceived versus those who did not. Multiple logistic regression models were used to compute odds ratios using age, sex, duration of union, and use of ART as covariates of uptake of preconception care. Statistical significance was set at $95 \%(p$ value $<0.05 \leq 0.05$ ). 


\section{Results}

Out of a total of $438 \mathrm{HIV}$ serodiscordant couples enrolled in the discordant couples' clinic, we included 219 (50\%) who expressed a desire to conceive in the analysis. Out of the 219 who expressed a desire to conceive only 91 (41.6\%) of couples took up preconception care services (Figure 1).

\subsection{Characteristics of the HIV Discordant Couples and Factors} Associated with Preconception Care Uptake. There was no significant difference in the comparison of the mean ages of males $(p=0.827)$ and females $(p=0.712)$ who took up preconception services compared to those who did not. Discordant couples who expressed a desire to conceive but did not take up the preconception services had a significantly ( $p=0.015)$ larger proportion of female HIV-positive partners. The median duration of the union/relationship was significantly $(p=0.005)$ longer among couples who chose not to take up the preconception services. The proportion of positive partners initiated on HAART was significantly ( $p=0.043)$ higher (100\% compared to $95.3 \%)$ among the partners in the preconception care group. Factors independently associated with uptake of preconception care were male sex of the HIV-positive partner union $(\mathrm{OR}=2.3$, CI $95 \%(1,1,5.0))$ and having a shorter duration of the discordant union $(\mathrm{OR}=0.92$, CI 95\% $(0.86,0.98))$ (Table 1).

\subsection{Use of HIV Prevention Methods among Couples Receiving} Preconception Care. All HIV-positive partners within the discordant couples' clinic received HAART. The prevention strategy with the highest uptake among the couples was the use of PrEP alongside HAART at $38.5 \%$, followed by HAART and AVI at $29.7 \%$. Within discordant unions where the HIV-infected partners were females, the use of PrEP was more common (64\%) compared to couples with male HIVpositive partners $(55.3 \%)$. The use of HAART only was reported among $20.9 \%$ of the couples. An interesting finding was a request for a referral for sperm wash services by approximately $8 \%$ of the couples where the male was HIV positive. These requests occurred despite couples receiving counselling on the reduced chances of transmission with optimal use of HAART and a confirmed viral load suppression $(<1000$ copies $/ \mathrm{ml}$ as per national guidelines) among the HIV-positive males. We referred these couples for sperm wash services provided at private clinics at the couples' cost. The smallest proportion (3.3\%) preferred timed unprotected intercourse (TUI) ( Figure 2).

3.3. Preconception Care Outcomes. Twenty seven (29.7\%) women accessing the preconception care conceived compared to $24(18.8 \%)$ who declined. We reported no seroconversions within the preconception care group. One uninfected partner seroconverted within the group that did not take up the services resulting in an HIV incidence rate of 0.19 per 100 person-years of follow-up. The overall couple retention was significantly $(p=0.004)$ higher among couples who accepted the services. Additionally, only three

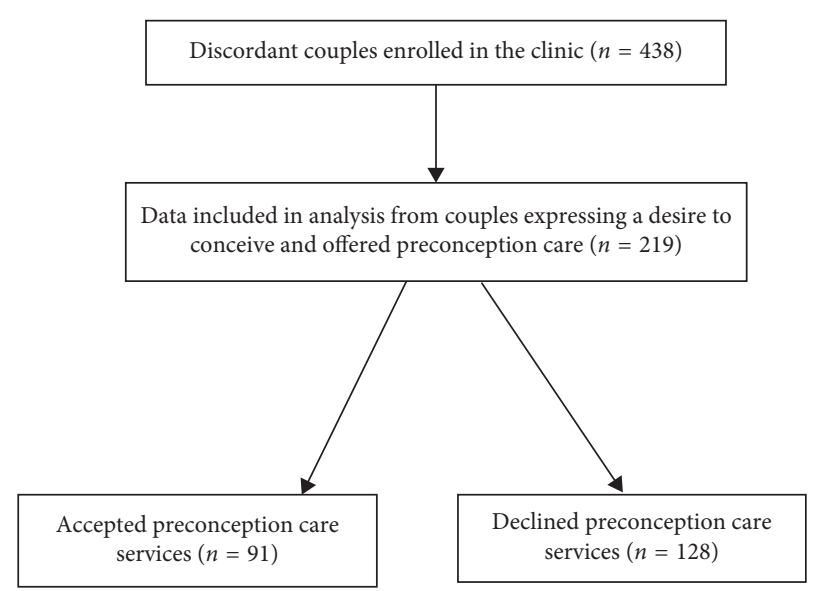

FIgURE 1: Flow diagram indicating sample of discordant couples' data included in the analysis, 133 for the study.

(2.3\%) partners in the preconception care cohort were lost to follow-up from the discordant couple care clinic compared with the 27 individuals (21\%) who declined preconception care (Table 2).

\section{Discussion}

We found an unexpected low uptake of preconception care services among couples who had expressed a desire to conceive. Among couples who took up the services, we reported a clear association between the duration of the union and increased service uptake. Our study also found that discordant couples with male HIV-positive partners were more likely to take up the services. An identified gap was the high attrition from discordant clinic follow-up among the HIV-negative partners.

The low uptake of preconception care services remains unexplained. However, HIV-related stigma, fear of unfamiliar medical procedures, and lack of information about the HIV prevention interventions among community members are barriers to uptake [10]. An insightful result from this study was that there was increased uptake of preconception care services among couples where males were the HIV-infected partners. We postulate that these two findings may be related to health information gaps and relationship power dynamics. The dominant role of the male partners' fertility desire in steering couples' fertility decisionmaking as is often seen in the pate societies, which may have been a contributing factor to driving the uptake [22].

There may have been inadequate health education on the benefits of preconception care during the introduction of preconception care to the discordant couples attending the clinic, leading to the overall poor uptake. However, conversely, couples may also have had prior knowledge of the higher risk of receptive penile-vaginal versus incentive penile-vaginal sexual intercourse $[15,22]$. This knowledge could also have contributed to lower uptake of the services mainly, where males were the HIV-negative partners as seen in this study. This finding if further interrogated would guide programmes on leveraging on male participation to improve uptake of preconception care. 
TABLE 1: Characteristics of couples expressing a desire to conceive who accepted preconception care services versus those who did not.

\begin{tabular}{|c|c|c|c|c|}
\hline \multirow{2}{*}{ Variable } & \multicolumn{2}{|c|}{ Preconception care services } & \multirow[t]{2}{*}{$P$ value } & \multirow{2}{*}{$\begin{array}{c}\text { Adjusted odds ratio } \\
95 \% \text { CI }\end{array}$} \\
\hline & Accepted $(n=91)$ & Not accepted $(n=128)$ & & \\
\hline Mean age in years of the female partner (SD) & $34.1(5.8)$ & $34.5(7.5)$ & 0.712 & $1.00(0.93-1.0)$ \\
\hline Mean age in years of the male partner (SD) & 39.7 (7.9) & $39.5(8.5)$ & 0.827 & $1.03(0.97-1.09)$ \\
\hline \multicolumn{5}{|l|}{ Sex of the positive partner, $n(\%)$} \\
\hline Female & $44(48.4)$ & $83(64.8)$ & & Ref \\
\hline Male & $47(51.6)$ & $45(35.2)$ & 0.015 & $2.3(1.1-5.0)$ \\
\hline Median duration of union in years (IQR) & $7(4-10)$ & $10(5-15)$ & 0.005 & $0.92(0.86-0.98)$ \\
\hline HIV-positive partner on HAART, $n(\%)$ & $91(100.0)$ & $122(95.3)$ & 0.043 & - \\
\hline
\end{tabular}

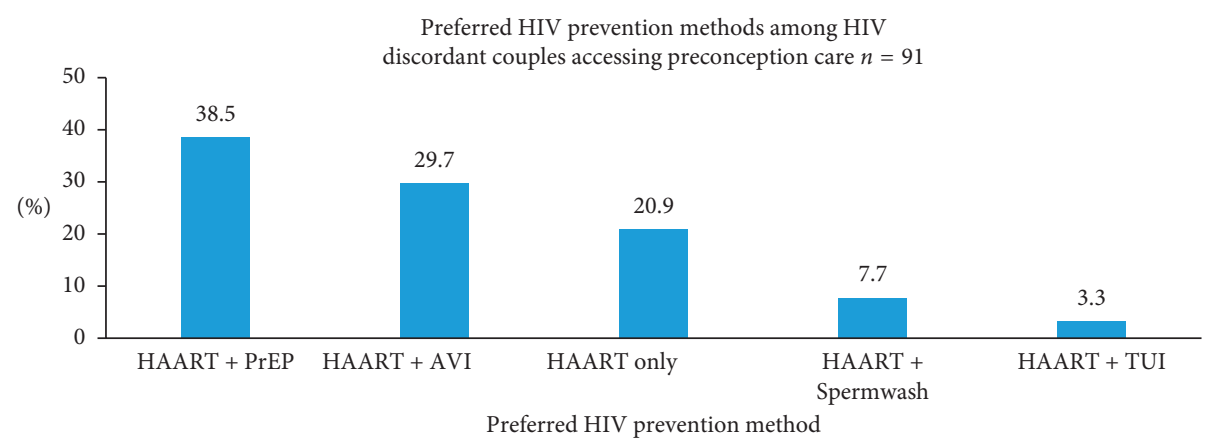

FIGURE 2: Discordant couple preferences for HIV prevention methods during preconception care.

TABle 2: Preconception care outcomes.

\begin{tabular}{|c|c|c|c|}
\hline \multirow{2}{*}{$\begin{array}{l}\text { Variable } \\
\text { Preconception care outcome, } n(\%)\end{array}$} & \multicolumn{2}{|c|}{ Preconception care services } & \multirow{2}{*}{$P$ value } \\
\hline & Accepted $(n=91)$ & Not accepted $(n=128)$ & \\
\hline Pregnancy & $27(29.7)$ & $24(18.8)$ & 0.060 \\
\hline No & $64(70.3)$ & $104(81.3)$ & \\
\hline \multicolumn{4}{|c|}{ Retention to discordant couple care, $n(\%)$} \\
\hline \multicolumn{4}{|c|}{ HIV-positive partner } \\
\hline Active & $90(98.9)$ & $117(91.4)$ & 206 \\
\hline Loss to follow-up & 0 & $3(2.3)$ & 0.091 \\
\hline Dead & 0 & $3(2.3)$ & \\
\hline Transfer out to other facility & $1(1.1 \%)$ & $5(3.9)$ & \\
\hline \multicolumn{4}{|l|}{ HIV-negative partner } \\
\hline Active & $83(91.2)$ & $96(75.0)$ & \\
\hline Loss to follow-up & $8(8.8)$ & $27(21.1)$ & \\
\hline Dead & 0 & 0 & 0.004 \\
\hline Transfer out to other facility & 0 & $5(3.9)$ & \\
\hline
\end{tabular}

The finding of the shorter duration of a discordant union as an independent determinant of increased uptake of preconception care services by the relatively young discordant couples was an exciting finding that is also demonstrated by other studies [20]. Additionally, the pregnancy rate of $9.6 \%$ in this study was within the range (9.6-16.6) reported in clinical trials which focussed on relatively young discordant couples $[17,21]$. These two findings further support existing literature that indicates that a desire for children in young couples (as evidenced by median age and short duration of the union) may be a significant potential contributor to HIV transmission [10, 21]. Though the difference in pregnancy rates between those who took up the services at $(29.7 \%)$ versus those who did not $(18.8 \%)$ was higher but not statistically different, information provided to couples who took up the services on fertility cycles and timing of ovulation most likely contributed to the relatively higher conception rates in this cohort.

With only one reported seroconversion event, the HIV incidence rate was lower than that reported among studies in Africa, in which the HIV incidence varied between 1.2 and 22 per 100 person-years with a median of 11.1 per 100 person-years [6]. However, we note that HIV incidence may be underreported in this study, taking into account the significant loss to follow-up reported among the couples who declined preconception. Nevertheless, this study demonstrates a decrease in HIV acquisition as a result of HAART, comparable to results from clinical trials among 
discordant couples in real-life program implementation $[13,16,17]$.

A limitation of this study was the use of retrospectively collected data which did not allow the inclusion of additional exposure variables that could have contributed to the outcomes. The study population may also be unique as a significant proportion had participated in clinical studies or expressed interest to attend discordant couples care clinics. Data on pregnancy outcomes of this cohort were challenging to obtain because once pregnancy is confirmed, they are referred to antenatal clinic and consequently, a high-risk clinic that follows up mothers and HIV-exposed infants.

This study, however, has the overarching strength of documenting the feasibility and successful implementation of preconception care interventions among discordant couples in the context of routine care in a low resource setting, providing new information outside the setting of the numerous research settings previously reported which focussed on the discordant couple.

\section{Conclusion}

We have demonstrated that in the context of routine HIV care and treatment for all couples, providing preconception care for discordant couples that express a desire to conceive is feasible and valuable. The introduction of preconception services should have robust health education and HIV prevention literacy component in order to improve couples understanding of the potential benefits. Strategies are required to mitigate the low HIV-infected partner retention enabling the actualisation of the full gains of preconception care. The introduction of conception care within discordant couple's routine care is essential and in future will play a significant role in the aversion of horizontal and vertical HIV transmission.

\section{Data Availability}

The data used to support the findings of this study are included in the supplementary information file.

\section{Conflicts of Interest}

The authors have no conflicts of interest to declare.

\section{Authors' Contributions}

NGK, S. M., J. K., and JOM made substantial contributions to the conception and design of the study. NGK, S. E., and K. N. acquired the data; NGK, K. M., and S. E. analysed the data. S. E., NGK, and K.M. drafted the manuscript. S. E., NGK, K. M., JOM, and J. K. critically reviewed and revised the manuscript; all authors approved the final draft of the manuscript.

\section{Acknowledgments}

The authors wish to acknowledge the management of Kenyatta National hospital comprehensive care centre led by
Dr Peter Muiruri. The authors would also like to thank Dr Elizabeth Kubo for editing the manuscript.

\section{Supplementary Materials}

Table 3 Study database. (Supplementary Materials)

\section{References}

[1] G. de Bruyn and C. Farquhar, "HIV-1-Discordant couples in sub-saharan Africa: explanations and implications for high rates of discordancy," Current HIV Research, vol. 5, no. 4, pp. 416-429, 2007.

[2] K. L. Dunkle, R. Stephenson, E. Karita et al., "New heterosexually transmitted HIV infections in married or cohabiting couples in urban Zambia and Rwanda: an analysis of survey and clinical data," The Lancet, vol. 371, no. 9631, pp. 2183-2191, 2008.

[3] H. Chemaitelly, I. Cremin, J. Shelton et al., "Distinct HIV discordancy patterns by epidemic size in stable sexual partnerships in sub-Saharan Africa," Sexually Transmitted Infections, vol. 88, no. 1, pp. 51-57, 2012.

[4] R. King, N. Wamai, K. Khana et al., "“Maybe his blood is still strong": a qualitative study among HIV-serodiscordant couples on ART in rural Uganda," BMC Public Health, vol. 12, no. 1, 2012.

[5] J. Beyeza-Kashesya, F. Kaharuza, F. Mirembe et al., "The dilemma of safe sex and having children: challenges facing HIV serodiscordant couples in Uganda," African Health Sciences, vol. 9, 2009.

[6] World Health Organisation, . Preconception Care: Maximising the Gains for Maternal and Child Health, World Health Organization, Geneva, Switzerland, 2013, www.who.int/maternal_child_adolescent/en\%0A@World.

[7] World Health Organisation, Consolidated Guidelines on the Use of Antiretroviral Drugs for Treating and Preventing HIV Infection-Recommendations for a Public Health Approach, World Health Organization, Geneva, Switzerland, 2015.

[8] J. M. McMahon, J. E. Myers, A. E. Kurth et al., "Oral preexposure prophylaxis (PrEP) for prevention of HIV in serodiscordant heterosexual couples in the United States: opportunities and challenges," AIDS Patient Care and STDs, vol. 28, no. 9, pp. 462-474, 2014.

[9] L. T. Matthews, J. M. Baeten, C. Celum et al., "Periconception pre-exposure prophylaxis to prevent HIV transmission: Benefits, risks, and challenges to implementation," AIDS, vol. 24, no. 13, pp. 1975-1982, 2010.

[10] R. J. Steiner, J. K. Dariotis, J. R. Anderson et al., "Preconception care for people living with HIV: recommendations for advancing implementation," AIDS, vol. 27, pp. S113-S119, 2013.

[11] O. Mmeje, S. Van Der Poel, M. Workneh et al., “Achieving pregnancy safely: perspectives on timed vaginal insemination among HIV-serodiscordant couples and health-care providers in Kisumu, Kenya," AIDS Care, vol. 27, no. 1, pp. 10-16, 2015.

[12] G. J. Wagner, K. Goggin, D. Mindry et al., "Correlates of use of timed unprotected intercourse to reduce horizontal transmission among Ugandan HIV clients with fertility intentions," AIDS and Behavior, vol. 19, no. 6, pp. 1078-1088, 2015.

[13] M. S. Cohen, Y. Q. Chen, M. McCauley et al., "Prevention of HIV-1 infection with early antiretroviral therapy," New England Journal of Medicine, vol. 365, no. 6, pp. 493-505, 2011. 
[14] A. Bhana, C. A. Mellins, I. Petersen et al., "The VUKA family program: piloting a family-based psychosocial intervention to promote health and mental health among HIV infected early adolescents in South Africa," AIDS Care, vol. 26, no. 1, pp. 1-11, 2014.

[15] M.-C. Boily, R. F. Baggaley, L. Wang et al., "Heterosexual risk of HIV-1 infection per sexual act: systematic review and metaanalysis of observational studies," The Lancet Infectious Diseases, vol. 9, no. 2, pp. 118-129, 2009.

[16] A. Miltz, A. N. Phillips, A. Speakman et al., "Implications for a policy of initiating antiretroviral therapy in people diagnosed with human immunodeficiency virus: the CAPRA research programme," Programme Grants for Applied Research, vol. 5, no. 18, pp. 1-40, 2017.

[17] J. M. Baeten, D. Donnell, P. Ndase et al., "Antiretroviral prophylaxis for HIV Prevention in heterosexual men and women," New England Journal of Medicine, vol. 367, no. 5, pp. 399-410, 2012.

[18] World Health Organisation, "Maternal and infant ARV prophylaxis to prevent MTCT for HIV-infected pregnant women who do not need treatment for their own health- antiretroviral drugs for treating pregnant women and preventing HIV infection in infants: recommendations for a public hea," NCBI Bookshelf, 2010.

[19] K. M. De Cock, G. W. Rutherford, and W. Akhwale, "Kenya AIDS indicator survey 2012," JAIDS Journal of Acquired Immune Deficiency Syndromes, vol. 2, pp. S3-12, 2014.

[20] B. L. Guthrie, R. Y. Choi, R. Bosire et al., "Predicting pregnancy in HIV-1-discordant couples," AIDS and Behavior, vol. 14, no. 5, pp. 1066-1071, 2010.

[21] J. Del Romero, M. B. Baza, I. Río et al., "Natural conception in HIV-serodiscordant couples with the infected partner in suppressive antiretroviral therapy: A prospective cohort study," Medicine, vol. 95, no. 30, Article ID e4398, 2016.

[22] J. Pintye, K. Ngure, K. Curran et al., "Fertility decisionmaking among Kenyan HIV-serodiscordant couples who recently conceived: implications for safer conception planning," AIDS Patient Care and STDs, vol. 29, no. 9, pp. 510516, 2015. 